\title{
Kondo Effect in ac Transport through Quantum Dots
}

\author{
Rosa López, ${ }^{1}$ Ramón Aguado, ${ }^{1,2}$ Gloria Platero, ${ }^{1}$ and Carlos Tejedor ${ }^{3}$ \\ ${ }^{1}$ Teoría de la Materia Condensada, Instituto de Ciencia de Materiales (CSIC) Cantoblanco, 28049 Madrid, Spain \\ ${ }^{2}$ Department of Applied Physics, Delft University of Technology, Lorentzweg 1, 2628CJ, Delft, The Netherlands \\ ${ }^{3}$ Física Teórica de la Materia Condensada, Universidad Autónoma de Madrid, Cantoblanco, 28049 Madrid, Spain
}

(Received 22 July 1998)

\begin{abstract}
A theory of the Kondo effect in quantum dots at zero temperature in the presence of arbitrarily intense ac potentials is presented. We generalize the Friedel-Langreth sum rule to take care of charge conservation and propose a consistent procedure to study a time-dependent Anderson Hamiltonian. The effect of the ac potential on both the quantum dot density of states and the linear conductance shows the importance of using a theory which describes intradot finite interaction and nonperturbative effects. [S0031-9007(98)07727-8]
\end{abstract}

PACS numbers: 72.15.Qm, 73.40.Gk, 73.50.Mx, 85.30.Vw

The competition between electron-electron interaction and quantum-mechanical hybridization between the delocalized electrons in a nonmagnetic metal and the unpaired electrons of a magnetic impurity leads to the Kondo effect [1]. It has been predicted [2-9] that the transport at low temperatures through a single level coupled to two reservoirs containing Fermi liquids [10] is governed by a Kondo-like singularity existing in the quasiparticle density of states (DOS) at the Fermi level $\epsilon_{F}$ of the reservoirs. This has been experimentally performed recently $[11,12]$ by means of a quantum dot (QD) coupled to two leads by tunneling barriers. When (i) the QD is small enough to have well-separated levels, (ii) the tunneling rate is such that the broadening of QD levels is typically 1 order of magnitude smaller than the QD charging energy, and (iii) the number of electrons within the QD is odd, then there is an unpaired electron which is free to form a singlet state with the electrons in the leads. This is the physics behind of the Kondo effect that is well described by the lowenergy excitations of the Anderson Hamiltonian for the unpaired electron while all of the other electrons are effectively described by a potential of the QD coupled to the leads. The excitations around $\epsilon_{F}$ are quasiparticles having a lifetime determined only by single particle properties without any many-body contribution. One electron at $\boldsymbol{\epsilon}_{F}$ becomes scattered by the dot suffering a phase shift which is proportional to the exact displaced charge $\delta n$. As a consequence of this Friedel-Langreth (FL) theorem [13], the conductance takes the value $G=\sin ^{2}(\pi \delta n) 2 e^{2} / h[2,3]$. The interest of the Kondo physics in QD resides in the possibility of externally controlling parameters as the on-site interaction $U$, the QD level $\epsilon_{0}$, or the broadening $\Gamma$ of the DOS, due to the dot-leads coupling [11,12]. It is also possible to study the Kondo singularity itself by applying a bias in order to mimic the QD DOS by the $d I / d V$ curve $[7,11,12]$.

In this Letter we address the following question: since the quasiparticles with large lifetime are those at $\epsilon_{F}$, what happens to the Kondo problem at zero temperature when there is a mixing of states with energies differing from
$\epsilon_{F}$ by a finite amount? In the case of QD, this can be actually made by means of an ac gate voltage [1417]. The problem is very interesting and far from trivial because the frequency range experimentally accessible (from $\sim 100 \mathrm{MHz}$ to $20 \mathrm{GHz}$ ) [18] is roughly that of the Kondo temperature $T_{K}=\sqrt{U \Gamma} \exp \left\{-\pi\left[\left|\epsilon_{F}-\epsilon_{0}\right|(U+\right.\right.$ $\left.\left.\left.\epsilon_{0}\right)\right] / \Gamma U\right\}$ (from a few $\mathrm{mK}$ to $\sim 1 \mathrm{~K}$ ) [12].

The aim of our Letter is to describe the QD DOS within a framework where charge conservation is taken into account in order to describe properly the Kondo singularity. Therefore, our first task is to generalize the FL sum rule to the case of a time-dependent situation. The Anderson Hamiltonian is solvable through Bethe ansatz [19] or quantum Monte Carlo methods [20], but a reliable and simple method to obtain dynamical properties at low temperatures in the whole range of interactions $(U / \Gamma)$ is not available. Previous efforts concentrated in the $U \rightarrow \infty$ limit where a noncrossing approximation $[5,14,17]$ can be made for high and intermediate temperatures. However, such approximations do not give the exact local Fermi-liquid properties when $T \rightarrow 0$ and cannot describe the transition from the weak-correlation to the strongcorrelation regime. On the other hand, finite $U$ perturbation theory $[6,21,22]$ describes properly the symmetric case but presents clear anomalies away from this special situation. Our method is an extension of those $[7,23]$ developed in the static case where an effective self-energy eliminates consistently the problems of the perturbative approaches.

The time-dependent Anderson Hamiltonian reads

$$
\begin{aligned}
H= & \sum_{k \in\{L, R\}, \sigma} \epsilon_{k} c_{k, \sigma}^{\dagger} c_{k, \sigma}+\sum_{\sigma}\left(\epsilon_{\sigma}+V_{\mathrm{ac}} \cos \omega_{0} t\right) d_{\sigma}^{\dagger} d_{\sigma} \\
& +\sum_{k \in\{L, R\}, \sigma} V_{k}\left(c_{k, \sigma}^{\dagger} d_{\sigma}+d_{\sigma}^{\dagger} c_{k, \sigma}\right)+U d_{\uparrow}^{\dagger} d_{\uparrow} d_{\downarrow}^{\dagger} d_{\downarrow} .
\end{aligned}
$$

$d_{\sigma}^{\dagger}$ creates an electron with spin $\sigma$ in the dot while $c_{k, \sigma}^{\dagger}$ creates it in the lead with energy $\epsilon_{k}$ ( $k$ labels the rest of the quantum numbers). The ac voltage with intensity $V_{\mathrm{ac}}$ 
and frequency $\omega_{0}$ modulates in time the relative position of the QD level $\epsilon_{\sigma}$ with respect to $\epsilon_{F}$. An eventual breakdown of the spin degeneracy would be represented by $\epsilon_{\sigma} \neq \epsilon_{-\sigma}$. The coupling $V_{k}$ between the QD and the leads produces a broadening $\Gamma^{L(R)}=-2 \operatorname{Im}\left[\sum_{s p}^{L(R)}(\epsilon+i \delta)\right]=$ $2 \pi \sum_{k \in L(R)}\left|V_{k}\right|^{2} \delta\left(\epsilon-\epsilon_{k}\right)$. The dc linear conductance at zero temperature deduced from a nonequilibrium Keldysh technique is [24]

$$
\mathcal{G}=\frac{e^{2}}{\hbar} \frac{\Gamma^{L}\left(\epsilon_{F}\right) \Gamma^{R}\left(\epsilon_{F}\right)}{\Gamma\left(\epsilon_{F}\right)} \sum_{\sigma} \rho_{\sigma}\left(\epsilon_{F}\right),
$$

where $\Gamma=\Gamma^{L}+\Gamma^{R} . G$ is related to the time averaged DOS at the QD $\rho_{\sigma}(\epsilon)=-\operatorname{Im}\left\langle A_{\sigma}(\epsilon)\right\rangle / \pi$ obtained from

$$
\left\langle A_{\sigma}(\epsilon)\right\rangle=\frac{\omega_{0}}{2 \pi} \int_{0}^{2 \pi / \omega_{0}} d t \int_{-\infty}^{t} d t_{1} G_{\sigma}^{r}\left(t, t_{1}\right) e^{i \epsilon\left(t-t_{1}\right)}
$$

where $G_{\sigma}^{r}\left(t, t_{1}\right)$ is the QD retarded Green's function. Coherent tunneling of one electron at $\epsilon_{F}$ through the QD with ac produces a phase shift of its wave function which is related, according to the FL rule, to the displaced charge for each spin which is related to the QD occupation $\left(\left\langle n_{\sigma}\right\rangle\right)$ and to the number of lead electrons with $\left(\left\langle n_{\sigma}^{l}\right\rangle\right)$ and without $\left(\left\langle n_{0, \sigma}^{l}\right\rangle\right)$ coupling to the QD:

$$
\eta_{\sigma}\left(\epsilon_{F}\right)=\pi\left(\left\langle n_{\sigma}\right\rangle+\left\langle n_{\sigma}^{l}\right\rangle-\left\langle n_{0, \sigma}^{l}\right\rangle\right) .
$$

The phase shift is related to $\left\langle A_{\sigma}(\epsilon)\right\rangle$ through the eigenvalues of the scattering $\mathcal{T}_{\sigma}$ matrix

$$
e^{2 i \eta_{\sigma}(\epsilon)}=1+\mathcal{T}_{\sigma}(\epsilon)=1-i \sqrt{\Gamma_{L} \Gamma_{R}}\left\langle A_{\sigma}(\epsilon)\right\rangle .
$$

The displaced charge in Eq. (4) can be obtained from the retarded Green's function as follows: (i) $\left\langle n_{\sigma}^{l}\right\rangle=-\operatorname{Im}\left\langle G_{k, \sigma}^{<}(t, t)\right\rangle$ and $\left\langle n_{\sigma}\right\rangle=-\operatorname{Im}\left\langle G_{\sigma}^{<}(t, t)\right\rangle$. (ii) $G^{<}$is related to the retarded Green's function built up using $G_{\sigma}^{r}\left(t, t_{1}\right)=G_{\sigma}^{r}\left(t-t_{1}\right) \exp \left[-\frac{i}{\hbar} \int_{t}^{t_{1}} d \tau \times\right.$ $\left.V_{\text {ac }} \cos \omega_{0} \tau\right]$, and (iii) application of the operational rules given by Langreth [25] for Keldysh contour integration. This process, together with Eqs. (4) and (5), gives

$$
\begin{aligned}
\operatorname{Im} \ln \left(\sum_{m=-\infty}^{\infty} J_{m}^{2}(\beta) G_{\sigma}^{r}\left(\epsilon_{F}-m \hbar \omega_{0}\right)\right)= & -\operatorname{Im} \sum_{m=-\infty}^{\infty} J_{m}^{2}(\beta) \int_{-\infty}^{\epsilon_{F}} d \epsilon \frac{\Gamma^{L}(\epsilon)+\Gamma^{R}(\epsilon)}{\Gamma\left(\epsilon-m \hbar \omega_{0}\right)} G_{\sigma}^{r}\left(\epsilon-m \hbar \omega_{0}\right) \\
& \times\left(1-\sum_{s=-\infty}^{\infty} J_{s}^{2}(\beta) \sum_{k} \frac{\left|V_{k}\right|^{2}}{\left[\epsilon-\epsilon_{k}-(m-s) \hbar \omega_{0}\right]^{2}}\right),
\end{aligned}
$$

where $G_{\sigma}^{r}(\epsilon)$ is the Fourier transform of the static retarded Green's function, $J_{m}$ is the Bessel function of order $m$, and argument $\beta=V_{\mathrm{ac}} / \hbar \omega_{0}$. Equation (6) reduces to the usual FL rule [13] for $V_{\mathrm{ac}} \rightarrow 0$. It establishes the charge neutrality constriction that must be verified by any approximation made to compute the retarded Green's function. This result implies a series of important consequences on the Kondo physics of the QD.

Some approximations are made to compute the retarded Green's function:

$$
G_{\sigma}^{r}(\epsilon)=\left[\epsilon-\epsilon_{\sigma}-\Sigma_{s p}(\epsilon)-\Sigma_{\sigma}(\epsilon)\right]^{-1} .
$$

Correlation effects are included in the self-energy $\Sigma_{\sigma}(\epsilon)$. In order to obtain $\Sigma_{\sigma}(\epsilon)$, we use an approximation which, in the static case [7,23], eliminates the pathologies of standard perturbation procedures. $\Sigma_{\sigma}(\epsilon)$ is calculated by an interpolation that gives correctly the limits $U / \Gamma \rightarrow 0$ and $\Gamma / U \rightarrow 0$ and has good analytic properties both in $\epsilon \rightarrow \epsilon_{F}$ and $\epsilon \rightarrow \pm \infty$ [7,23]:

$$
\Sigma_{\sigma}(\epsilon)=U\left\langle n_{-\sigma}\right\rangle+\frac{\left\langle n_{-\sigma}\right\rangle\left(1-\left\langle n_{-\sigma}\right\rangle\right)}{\left\langle n_{0,-\sigma}\right\rangle\left(1-\left\langle n_{0,-\sigma}\right\rangle\right)} \Sigma_{\sigma}^{(2)}(\epsilon)\left(1-\frac{\left[\left(1-\left\langle n_{-\sigma}\right\rangle\right) U+\epsilon_{\sigma}+\mu_{\sigma}\right]}{\left\langle n_{0,-\sigma}\right\rangle\left(1-\left\langle n_{0,-\sigma}\right\rangle\right) U^{2}} \Sigma_{\sigma}^{(2)}(\epsilon)\right)^{-1} .
$$

$\left\langle n_{0, \sigma}\right\rangle$ is the mean field (MF) QD occupation and $\Sigma_{\sigma}^{(2)}(\epsilon)$ is the second order self-energy used in previous finite $U$ calculations [6,7,21-23]. $\quad \mu_{\sigma}$ is a shift of the QD level to an effective value determined self-consistently together with $\left\langle n_{\sigma}\right\rangle$ with the constriction of charge neutrality given by Eq. (6). Once these parameters are determined, spectral and transport properties are calculated from $G_{\sigma}^{r}(\epsilon)$.

Hereafter, we consider $\Gamma^{L}=\Gamma^{R}=\Gamma, \epsilon_{\sigma}=\epsilon_{-\sigma}=$ $\epsilon_{0}$, and $\epsilon_{F}=0$. All of our calculations are performed with parameters in the range of the experiments already available $[11,12]$ in order to look for predictions on possible future experiments. First of all, we analyze the effect of the ac field on the time averaged DOS that can be measured by means of the $d I / d V$ curve of the QD as reported in the static case [11,12]. Figure 1 shows the DOS for a case with $\epsilon_{0} \neq-U / 2$. Results obtained from both Eq. (8) for $\Sigma_{\sigma}(\epsilon)$ (continuous lines) and for the self-energy up to sec- ond order (dashed lines) are shown. The self-consistency for the self-energy enhances the particle-hole asymmetry in both the static (Fig. 1a) and high frequency (Fig. 1d) cases. The lowest frequency (Fig. 1b) corresponds to an adiabatic modulation of the level $\left(\hbar \omega_{0}<\Gamma\right)$ which quenches the Kondo effect on the time averaged DOS. The two small peaks of the DOS correspond to the MF (lateral peaks) solutions separated by the extreme values of the harmonic potential. Figure 1c corresponds to the case $\beta=2$ where the symmetric shape is recovered due to a transfer of spectral weight from the Kondo peak to the MF peaks and their satellites. Figure 1d shows the richest configuration with a strong asymmetry. This is better observed in Fig. 2 where the evolution of the DOS with $V_{\mathrm{ac}}$ is analyzed. In the case of $V_{\mathrm{ac}}=0.4$, one observes three types of peaks. Those labeled as $a$ correspond to the Kondo peak and its ac replicas. Those labeled as $b$ correspond to the low-energy MF 


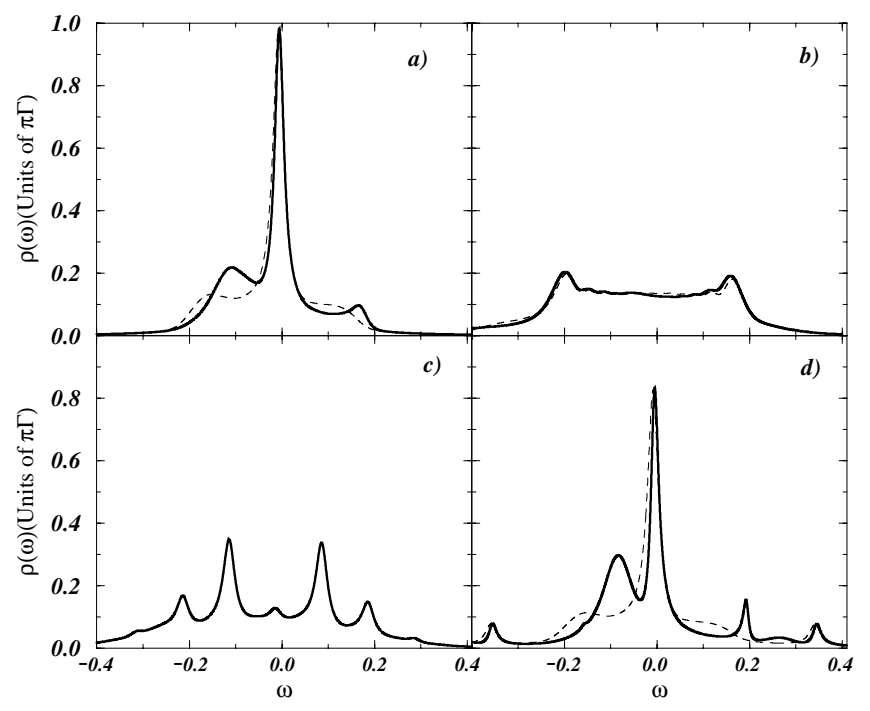

FIG. 1. Time averaged DOS with $V_{\mathrm{ac}}=0.2$ for the asymmetric configuration $U=0.2, \epsilon_{0}=-0.15$, and $\Gamma=0.025$. (a) without ac, (b) $\hbar \omega_{0}=0.01$, (c) $\hbar \omega_{0}=0.1$, (d) $\hbar \omega_{0}=0.35$. Solid lines are calculated with the interpolation procedure for the self-energy $\Sigma_{\sigma}(\epsilon)$ [Eq. (8)] and dotted lines with the selfenergy up to second order.

peak for $V_{\mathrm{ac}}=0$ and its ac replicas. These peaks evolve to higher energies and become narrower with increasing $\beta$. Finally, the peaks labeled as $c$ correspond to the highenergy MF peak for $V_{\mathrm{ac}}=0$ and its ac replicas. Once again, the evolution with $\beta$ is similar to that of peaks $b$. Moreover, with increasing intensity, we observe a decrease of both the height and the width of the peak at the Fermi level. These effects are a consequence of the appearance of increasing new peaks and the necessary conservation of the total spectral weight. The reduction of the height affects the conductance as discussed below while that of the width implies a change in $T_{K}$. The behavior of the spectrum is not trivial (e.g., occupations change with $\beta$ ), and clearly different to that obtained in a single particle picture of ac-assisted tunneling, where only simple replicas of the static DOS would appear.

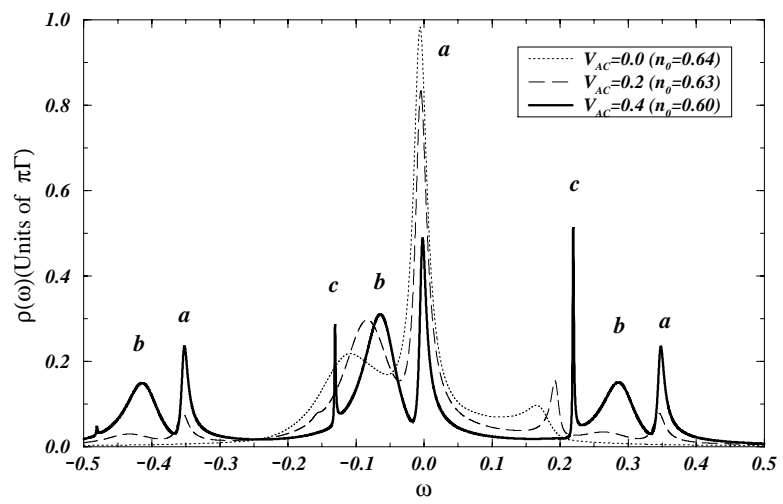

FIG. 2. Time averaged DOS with $\hbar \omega_{0}=0.35$ for the asymmetric configuration $U=0.2, \epsilon_{0}=-0.15$, and $\Gamma=0.025$ (as in Fig. 1) and different intensities $V_{\mathrm{ac}}$. The labels $a, b$, and $c$ are discussed in the text.
The enormous changes in the DOS when the occupation varies affect strongly the conductance. Therefore, the effect of the ac potential on $G$ cannot be described now by a single-particle model of ac-assisted tunneling through a discrete state [26]. Figure 3 shows the ac effects on $G$. At zero temperature and in the absence of ac potential [2-4] $G$ has a broad peak of width given by $U$ centered around $\epsilon_{0}=-U / 2$ and reaches the maximum value of $2 e^{2} / h$ [27]. Figure 3 shows a reduction of the central peak (still centered around $\epsilon_{0}=-U / 2$ ) and the appearance of symmetrically located satellites due to the ac. The position of the conductance satellites is inferred from the following argument: the DOS (left inset of Fig. 3) has a peak when the renormalized level of the QD is equal to $\hbar \omega_{0}$. It has an ac satellite at $\epsilon_{F}$ (dashed line). This gives a large contribution to the conductance. For low $\beta$, the level renormalization is small so that the peak in $G$ is located at $\epsilon_{0}-\hbar \omega_{0} \simeq 0$. Since the conductance is symmetric with respect to $-U / 2$, that satellite has a companion at $\epsilon_{0} \simeq-U-\hbar \omega_{0}$. So, the separation between satellites is $U+2 \hbar \omega_{0}$ as observed in Fig. 3 for $V_{\mathrm{ac}}=0.2$. When the ac intensity increases, the QD level renormalization increases and the reasoning, still exactly valid for the renormalized level, gives only approximately the separation in $\epsilon_{0}$ between satellites. In the same range of values for $U$ and $\Gamma$, the results are similar for $\hbar \omega_{0}=$ $U / 2, U / 4$, and $3 U / 20$. It must be stressed that, whereas the satellites in $G$ mark the ac-assisted transition from the empty orbital regime to the mixed valence regime (inset at the left side of Fig. 3), the main broad peak corresponds to tunneling in the Kondo regime (inset at the right side of Fig. 3). This behavior in the conductance is different from that predicted by a MF theory, where satellites at $-\hbar \omega_{0}$ and $\hbar \omega_{0}-U$ also appear. The lack of these

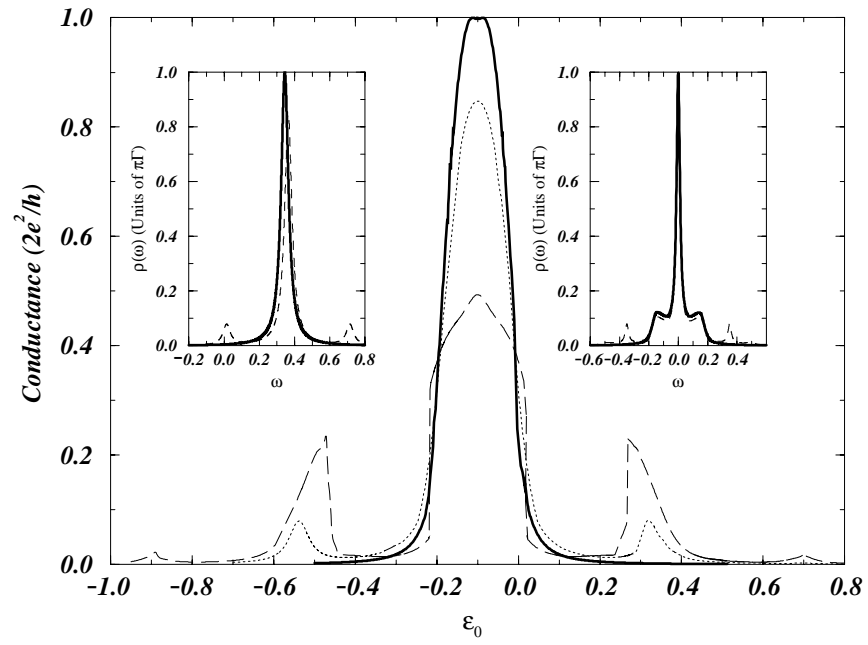

FIG. 3. Linear conductance with $\hbar \omega_{0}=0.35, U=0.2$, and $\Gamma=0.025$ for $V_{\mathrm{ac}}=0$ (continuous line), $V_{\mathrm{ac}}=0.2$ (dotted line), and $V_{\mathrm{ac}}=0.4$ (dashed line). The insets show the DOS with (dashed lines) and without (continuous lines) ac at the empty orbital (left side, $\epsilon_{0}=0.35$ ) and Kondo (right side, $\left.\epsilon_{0}=-0.1\right)$ regimes. 


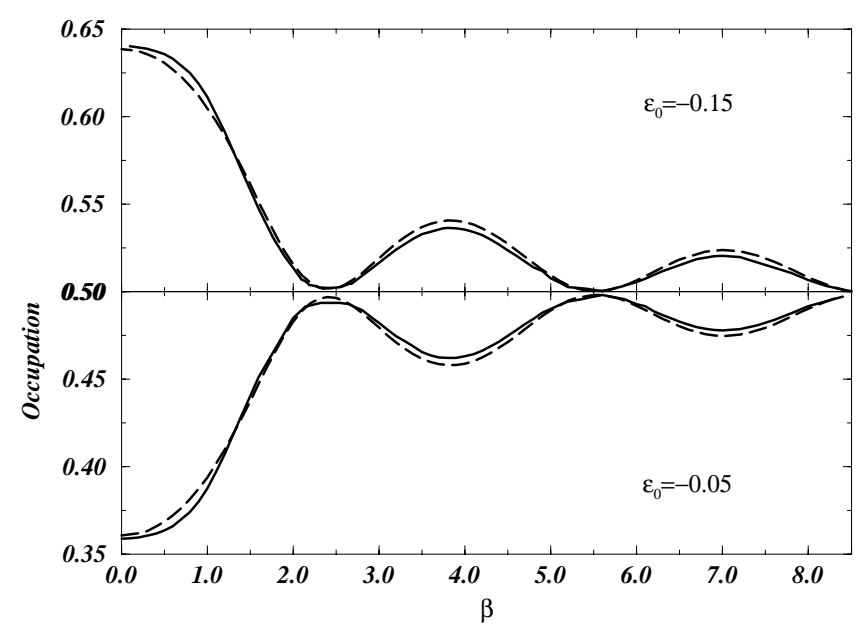

FIG. 4. Occupation $\left\langle n_{\sigma}\right\rangle$ of the QD as a function of $\beta$ for $U=0.2$ and $\hbar \omega_{0}=0.35$. Results with the self-energy up to second order (dashed lines) and with Eq. (8) (continuous lines) are shown for $\epsilon_{0}=-0.15$ (curves above) and $\epsilon_{0}=-0.05$ (curves below).

peaks is due to the different nature of the DOS when passing from the Kondo regime to the empty orbital regime (single peaked DOS centered at $\epsilon_{0}$ ) through the mixed valence one where the DOS has a single peak centered in a strongly renormalized position $[1,19]$. Our general result which depends on $U$ contrasts with the results of Ref. [14]. They used a model with $U \rightarrow \infty$ and found peaks of halfwidth $\Gamma$ in the conductance separated by $\hbar \omega_{0}$, similar to previously reported MF ac-assisted tunneling. Another very interesting result drawn from Fig. 3 is the nonlinear reduction of the central peak of the conductance when $V_{\mathrm{ac}}$ increases. Since the whole behavior of $G$ with $\omega_{0}$ is rather different from the linear dependence expected from a MF model, our result constitutes a proof of the importance of a finite $U$ charge conserving theory for the ac Kondo effect.

Finally, we pay attention to the effect of the ac potential on the occupation of the QD level. We show in Fig. 4 the charge in the dot in terms of $\beta$. Results are given for two different values of $\epsilon_{0}$ corresponding to initial occupations clearly above and below $1 / 2$ (per spin), respectively. For increasing $\beta,\left\langle n_{\sigma}\right\rangle$ tends to $1 / 2$ in a damped oscillatory way. For $\beta$ such that $J_{0}(\beta) \rightarrow 0$, the peak at $\epsilon_{0}$ in the DOS is quenched and its spectral weight transferred to the ac satellites. With the chosen parameters, half of the satellites are above/below zero (with the same intensities), so that the QD occupation tends to 1/2.

In conclusion, we have studied the ac Kondo effect in QD. We extend the FL sum rule to consider finite $U$ as well as charge conservation. Our main findings are (i) changes in the shape and position of the peaks in the DOS in the presence of the ac potential, and (ii) variation of the DOS at $\epsilon_{F}$ and, consequently, of the linear conductance which has new features with respect to the usual MF theory. These are finite- $U$ many-body effects. With regard to the possibility of experimentally observing the features described here, narrowing of peaks at the DOS due to ac effects are going to reduce $T_{K}$. However, there is still margin for the measurements: for instance, in the mixed valence regime, $T_{K} \sim \Gamma \sim 1 \mathrm{~K}$ in available experiments [12] (without ac) while temperatures significantly lower can be routinely achieved.

We acknowledge A.P. Jauho, L. Glazman, L.P. Kouwenhoven, A. Martin-Rodero, and J. J. Palacios for helpful discussions. This work was supported in part by the MEC of Spain under Contracts No. PB96-0875 and No. PB96-0085, and by the EU via Contract No. FMRXCT98-0180.

[1] A. C. Hewson, The Kondo Problem to Heavy Fermions (Cambridge University Press, Cambridge, 1993).

[2] T. K. Ng and P. A. Lee, Phys. Rev. Lett. 61, 1768 (1988).

[3] L. I. Glazman and M. E. Raikh, JETP Lett. 47, 452 (1988).

[4] A. Kawabata, J. Phys. Soc. Jpn. 60, 3222 (1991).

[5] Y. Meir et al., Phys. Rev. Lett. 66, 3048 (1991); 70, 2601 (1993).

[6] S. Hershfield et al., Phys. Rev. Lett. 67, 3720 (1991).

[7] A. L. Yeyati et al., Phys. Rev. Lett. 71, 2991 (1993).

[8] N. S. Wingreen and Y. Meir, Phys. Rev. B 49, 11040 (1994).

[9] Y. Wan et al., Phys. Rev. B 51, 14782 (1995).

[10] Local Fermi-liquid theory breaks down when the number of channels exceeds twice the localized spin. The behavior is that of a non-Fermi liquid. See, e.g., N. Andrei and C. Destri, Phys. Rev. Lett. 52, 364 (1984).

[11] D. Goldhaber-Gordon et al., Nature (London) 391, 156 (1998); LANL archives, cond-mat/9807233.

[12] S. M. Cronenwett et al., Science 281, 540 (1998).

[13] D. C. Langreth, Phys. Rev. 150, 516 (1966).

[14] M. H. Hettler and H. Schoeller, Phys. Rev. Lett. 74, 4907 (1995).

[15] T. K. Ng, Phys. Rev. Lett. 76, 487 (1996).

[16] A. Schiller and S. Hershfield, Phys. Rev. Lett. 77, 1821 (1996).

[17] P. Nordlander et al., LANL archives, cond-mat/9801241.

[18] L. P. Kouwenhoven et al., Phys. Rev. Lett. 73, 3443 (1994).

[19] A. M. Tsvelik and P. B. Wiegmann, Adv. Phys. 32, 453 (1983).

[20] R. M. Fye and J. E. Hirsch, Phys. Rev. B 38, 433 (1988).

[21] K. Yosida and K. Yamada, Prog. Theor. Phys. Suppl. 46, 224 (1970).

[22] B. Horvatic and V. Zlatic, Solid State Commun. 54, 957 (1985).

[23] H. Kajueter and G. Kotliar, Phys. Rev. Lett. 77, 131 (1996).

[24] A. P. Jauho et al., Phys. Rev. B 50, 5528 (1994).

[25] D.C. Langreth, in Linear and Nonlinear Electron Transport in Solids, edited by J.T. Devreese and V.E. Van Doren, NATO ASI, Ser. B, Vol. 17 (Plenum, New York, 1976).

[26] P. K. Tien and J. Gordon, Phys. Rev. 129, 647 (1963).

[27] When $T$ increases, the center of the peak is quenched and two peaks, at $\epsilon_{0}=-U$ and $\epsilon_{0}=0$, become apparent. They would correspond to the Coulomb Blockade peaks experimentally observed in QD for $T>T_{K}[11,12]$. 